
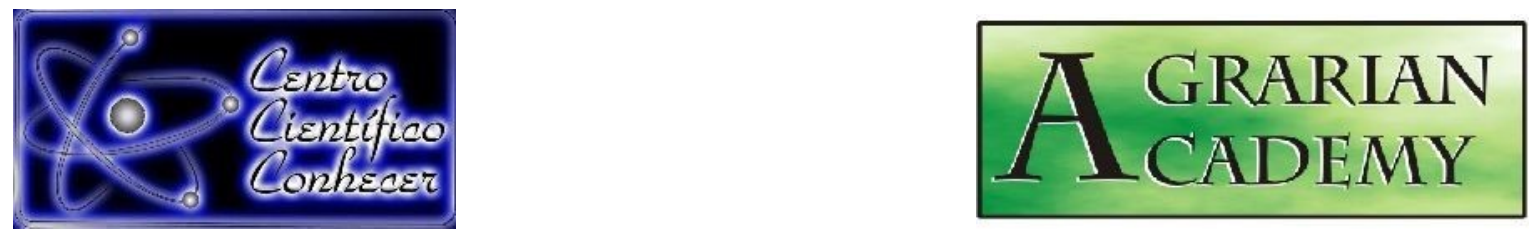

\title{
MODELAGEM LINEAR GENERALIZADA PARA DESCREVER A RELAÇÃO HIPSOMÉTRICA DE Eucalyptus saligna Smith.
}

Gabriel Fernandes Bueno ${ }^{1 *}$; Emanuel Arnoni Costa2; Guilherme Estevam Tanaka1; Ariane de Andrade ${ }^{1}$; Cristine Tagliapietra Schons ${ }^{3}$

\author{
${ }^{1}$ Acadêmico(a) do Curso de Engenharia Florestal, Universidade Federal de \\ Uberlândia, MG, Brasil, *(gabrielfbueno@outlook.com); \\ ${ }^{2}$ Prof. Dr. do Curso de Engenharia Florestal, Universidade Federal de Uberlândia, \\ MG, Brasil; \\ ${ }^{3}$ Doutoranda do Programa de Pós-graduação em Engenharia Florestal, \\ Universidade Federal de Santa Maria, RS, Brasil.
}

Recebido em: 01/04/2020 - Aprovado em: 10/06/2020 - Publicado em: 30/07/2020 DOI: 10.18677/Agrarian_Academy_2020a4

\begin{abstract}
RESUMO
A medição de alturas em povoamentos florestais é uma atividade onerosa, dependente da disponibilidade de recursos financeiros, tempo e pessoal qualificado e, portanto, a possibilidade de predição dessa variável traz inúmeras vantagens. Desta forma, o presente estudo teve por objetivo comparar e selecionar equações hipsométricas para Eucalyptus saligna Smith. utilizando a abordagem de Modelos Lineares Generalizados (MLG) a fim de gerar estimativas precisas de altura para a espécie. Foram testados quatro modelos de altura [h] em função do diâmetro [d], com variáveis independentes na forma: linear [d], quadrática [ $\left.\mathrm{d}^{2}\right]$, inversa [1/d] e logarítmica [In(d)], e a variável resposta [h] por meio da distribuição Gamma. Duas funções de ligação (F.L.) foram testadas: identidade $[\mu]$ e logarítmica $[\ln (\mu)]$. O teste de ShapiroFrancia evidenciou que a distribuição de frequência das alturas não apresentou normalidade, ressaltando a importância do uso da técnica de MLG, em especial a distribuição Gamma, devido a sua maior flexibilidade a dados assimétricos. A F.L. identidade $[\mu]$ foi a mais adequada, e a equação 3 , com a variável independente inversa [1/d], apresentou os melhores resultados de qualidade de ajuste, com coeficiente de determinação $\left[R^{2}=0,367\right]$, raiz do erro quadrático médio [RMSE = $1,64 \mathrm{~m}]$ e o critério de informação de Akaike [AIC =115,9]. A validação dessa equação, segundo o teste de qui-quadrado $\left(X^{2}\right)$, não apresentou diferenças significativas entre os dados observados e estimados. Considerando a sua flexibilidade, o MLG é uma importante técnica a ser aplicada na modelagem da altura em plantios de eucalipto com acuradas estimativas.
\end{abstract}

PALAVRAS-CHAVE: dendrometria, inventário florestal, relação altura-diâmetro. 


\title{
GENERALIZED LINEAR MODELS TO DESCRIBE THE HIPSOMETRIC RELATIONSHIP OF Eucalyptus saligna Smith.
}

\begin{abstract}
Height measurement in forest stand is an onerous activity, that depends on the availability of financial resources, time, and qualified personnel, therefore, the possibility of predicting this variable has numerous advantages. In this sense, the present study aimed to compare and select hipsometric equations for Eucalyptus saligna Smith. using the Generalized Linear Models (GLM) approach to generate accurate height estimative for the specie. Four height models [h] were tested according to the diameter [d], with independent variables in form: linear [d], quadratic $\left[\mathrm{d}^{2}\right]$, inverse [1/d] and logarithmic [In(d)], and the response variable [h] through the Gamma distribution. Two link functions (L.F) were tested: identity $[\mu]$ and logarithmic $[\ln (\mu)]$. The Shapiro-Francia test proved the non-normality of the data, emphasizing the importance of using GLM, especially the Gamma distribution due to its greater flexibility to asymmetric data. The identity $[\mu]$ L.F was the most adequate, whose equation 3 with inverse [1/d] independent variable presented the best fit quality results, with determination coefficient $\left[R^{2}=0.367\right.$ ], root of the mean square error [RMSE $=1.64$ ], Akaike information criterion [AIC $=115.9]$. The validation of this equation, according to the chi-square test $\left(X^{2}\right)$, showed no significative differences between the observed and estimate data. Considering its greater flexibility, GLM is an important technique to be applied in height modeling in eucalyptus plantations with accurate estimates.
\end{abstract}

KEYWORDS: dendometry, forest inventory, height-diameter relationship.

\section{INTRODUÇÃO}

O setor florestal ganha espaço na representatividade do PIB e importância na economia mundial e brasileira, já que $59 \%$ da área territorial do Brasil é ocupada por florestas, representando $12 \%$ de todo planeta (FAO, 2015). As principais culturas plantadas no país atualmente são: eucalipto com 7.401.334 hectares, e pinus com 2.030.419 hectares (IBGE, 2016).

Apenas no ano de 2018, o Brasil teve um superavit de US\$11,4 bilhões, representando um avanço de aproximadamente $26 \%$. Este número representa cerca de $5,2 \%$ das exportações brasileiras, beneficiando a população e sociedade com 3,8 milhões de empregos, diretos ou indiretamente pelo efeito renda. As florestas plantadas geram 6,9\% no PIB industrial (IBÁ, 2017).

Para o planejamento da produção florestal comumente são realizados inventários florestais, nos quais a medição da variável altura do povoamento é de grande importância para a estimativa do volume e qualificação da produtividade em determinado local (RIBEIRO et al., 2010). Porém, é comum que ocorram diversos erros durante as medições, sejam sistemáticos ocasionados pelo instrumento de medição ou até mesmo do próprio operador que não apresenta conhecimento técnico específico para execução da tarefa ou dificuldade de visibilidade da copa por condições adversas, além disso, a medição dessa variável demanda maior tempo, elevando os custos dos inventários florestais (SOARES et al., 2017).

Dessa forma, é possível recorrer ao uso de modelos hipsométricos, isto é, equações ajustadas que descrevem a relação altura-diâmetro da árvore (SANQUETTA et al., 2014). As relações hipsométricas sofrem influência de fatores como a espécie, idade, densidade, sítio e posição sociológica (COSTA et al., 2014; 
RETSLAFF et al., 2015; NICOLETTI et al., 2016; CERQUEIRA et al., 2019). Portanto, é necessário utilizar métodos eficientes para predição da altura, uma vez que o erro poderia subestimar ou superestimar a produção, o que não é desejado.

Uma maneira de descrever a relação hipsométrica é através de modelos lineares generalizados (MLG), que são uma adaptação do clássico modelo linear: $Y=X \beta+\varepsilon$, cujo $X$ é denominado como uma matriz de dimensão $\eta \times p$ de diferenciação do modelo (em geral é uma matriz de covariáveis com um primeiro vetor unitário), que associase a um vetor $\beta=\left(\beta_{1}, \ldots, \beta_{n}\right)^{\top}$, correspondendo aos coeficientes, e $\varepsilon$ é um vetor com os erros de distribuição aleatória (LINDSEY, 1997).

A grande vantagem da utilização de MLG está relacionada à análise de dados em que a suposição de normalidade não é plausível, possibilitando modelar variáveis de interesse que permite incorporar dados que exibem assimetria, dados de natureza discreta ou contínua e que são restritos a um intervalo do conjunto dos reais (CORDEIRO; DEMÉTRIO, 2013).

A utilização do MLG permite estender o conceito de homogeneidade entre grupos para dados da família exponencial e obter um leque da distribuição da variável resposta de uma amostra aleatória de $\eta$ observações independentes, sendo modelos aleatórios ou sistemáticos (CORDEIRO; DEMÉTRIO, 2013). O melhor modelo é aquele que apresenta melhor performance segundo os critérios de qualidade de ajuste testados. Convém ressaltar, que os modelos a serem comparados devem usar as mesmas observações, variáveis explicativas $\left(X_{1}, X_{2}, X_{n}\right)$ e variável resposta $(Y)$ (BAUTISTA, 2014).

Desta forma, o presente estudo teve por objetivo comparar e selecionar equações hipsométricas para Eucalyptus saligna Smith. usando a abordagem de Modelos Lineares Generalizados (MLGs) a fim de gerar estimativas precisas de altura para a espécie.

\section{Caracterização da área}

\section{MATERIAL E MÉTODOS}

O presente estudo foi realizado em uma área de $\approx 0,2$ ha de Eucalyptus saligna Smith. localizada no município de Rosário do Sul, RS. O clima na região é subtropical úmido do tipo Cfa, conforme a classificação de Köppen, com altitude de 145,3 m, temperatura média anual de $18,0{ }^{\circ} \mathrm{C}$ e a precipitação média anual de $1820 \mathrm{~mm}$ (ALVARES et al., 2013).

\section{Levantamento dos dados}

$\mathrm{Na}$ área foram medidas 165 árvores com 84 meses de idade, em espaçamento de $3,0 \times 2,0 \mathrm{~m}$. O diâmetro à altura do peito (d) foi medido com suta e a altura total (h) com o hipsômetro Vertex III. O conjunto de dados com pares de h-d foi subdividido de modo aleatório em dois grupos: i) usado para o ajuste dos modelos hipsométricos (115 árvores $\cong 70 \%$ ); e ii) usado para a validação dos modelos ajustados (50 árvores $\cong$ $30 \%)$.

\section{Modelo Linear Generalizado (MLG)}

Os quatro modelos a seguir foram testados para descrever a relação hipsométrica: 
1. $-h=\beta_{0}+\beta_{1} \times d+\varepsilon_{i}$

2. $-h=\beta_{0}+\beta_{1} \times d^{2}+\varepsilon_{i}$;

3. $-h=\beta_{0}+\beta_{1} \times 1 / d+\varepsilon_{i}$;

4. $-h=\beta_{0}+\beta_{1} \times \ln (d)+\varepsilon_{\text {i }}$.

Em que: $h=$ altura total, em $\mathrm{m} ; \mathrm{d}=$ diâmetro à altura do peito, em $\mathrm{cm} ; \beta_{0}$ e $\beta_{1}=$ coeficientes de regressão estimados; $\varepsilon_{i}=$ erro aleatório.

Para o ajuste dos modelos foi aplicada a abordagem de Modelos Lineares Generalizados (MLGs) (FOX, 2015). Os MLGs são uma variação dos modelos tradicionais, podendo ser aplicados para dados na forma de contagem, binárias e categóricas, com aplicação mais ampla. Eles são formados através da combinação de componentes aleatórios e sistemáticos (MÜLLER, 2012).

Segundo Cordeiro e Demétrio (2013) os MLG são caracterizados por:

(i) Componente aleatório: variáveis independentes aleatórias $Y_{1}, \ldots, Y_{n}$, com média $\mu_{\mathrm{i}}$. Sendo:

$$
\mathrm{E}\left(\mathrm{Y}_{\mathrm{i}}\right)=\mu_{\mathrm{i}}, \quad \mathrm{i}=1, \ldots, \mathrm{n}
$$

(ii) Componente sistemático: um conjunto de variáveis explanatórias $\mathrm{X}_{1}, \ldots, \mathrm{X}_{\mathrm{m}}$ e preditas linearmente:

$$
\eta=X \beta
$$

(iii) Função de ligação: conecta o componente aleatório ao componente sistemático.

$$
g\left(\mu_{\mathrm{i}}\right)=\eta_{\mathrm{i}}
$$

No presente estudo, a variável resposta foi avaliada por meio da distribuição Gamma com as funções de ligação (F.L.) identidade $[\mu]$ e logarítmica $[\ln (\mu)]$. Inicialmente, os dados foram analisados quanto à normalidade da distribuição de frequência da variável resposta por meio do teste de Shapiro-Francia à $5 \%$ de significância.

\section{Estatísticas de qualidade dos modelos}

A eficiência dos modelos ajustados foi baseada nas seguintes estatísticas de qualidade (MOSER; OLIVEIRA, 2017):

1. Coeficiente de determinação:

$$
R^{2}=1-\left[\frac{\sum_{i=1}^{n}\left(Y_{i}-\widehat{Y}\right)^{2}}{\sum_{i=1}^{n}\left(Y_{i}-\bar{Y}\right)^{2}}\right]
$$


2. Raiz do erro médio quadrático:

$$
\operatorname{RMSE}=\sqrt{(n-p)^{-1} \sum_{i=1}^{n}\left(Y_{i}-\widehat{Y}\right)^{2}}
$$

3. Critério de informação de Akaike:

$$
A I C=n \cdot \ln \left(\sum_{i=1}^{n}\left(\mathrm{Y}_{\mathrm{i}}-\widehat{\mathrm{Y}}\right)^{2}\right)-n \cdot \ln (n)+2 p
$$

4. Critério de informação de Akaike ponderado:

$$
w_{i}(\mathrm{AIC})=\frac{\mathrm{e}^{-\frac{1}{2} \Delta_{\mathrm{i}}(\mathrm{AIC})}}{\sum_{\mathrm{k}=1}^{\mathrm{k}} \mathrm{e}^{-\frac{1}{2} \Delta_{\mathrm{k}}(\mathrm{AIC})}}
$$

5. Resíduos em porcentagem:

$$
\text { Resíduos } \%=\frac{\mathrm{Y}_{\mathrm{i}}-\widehat{\mathrm{Y}}}{\mathrm{Y}_{\mathrm{i}}} .100
$$

Em que: $\mathrm{Y}=$ altura observada, em $\mathrm{m} ; \widehat{\mathrm{Y}}=$ altura estimada, em $\mathrm{m} ; \overline{\mathrm{Y}}=$ média das alturas, em $\mathrm{m} ; \mathrm{n}=$ número de observações; $\mathrm{p}=$ número de coeficientes; $\Delta_{i}(\mathrm{AIC})=$ $\mathrm{AlC}_{\mathrm{i}}-\min (\mathrm{AIC})$ e $\mathrm{k}=$ número de modelos a serem comparados.

A validação do melhor modelo foi analisada pelo teste Qui-Quadrado $\left(x^{2}\right)$ a $1 \%$ de probabilidade para comparar a altura real com a altura estimada.

\section{Análises estatísticas}

As estatísticas foram calculadas usando o software R ( $R$ CORE TEAM, 2020), a edição e desenvolvimento de gráficos com o Microsoft Excel 2019 (MICROSOFT CORPORATION, 2019).

\section{RESULTADOS}

Os pares de dados de $h-d$ subdivididos para o ajuste dos modelos hipsométricos apresentaram uma amplitude diamétrica de 16,2 a $22,2 \mathrm{~cm}$, com média de $19,1 \mathrm{~cm}$. O valor médio das alturas foi de $26,0 \mathrm{~m}$, com mínimo de $18,7 \mathrm{~m}$ e máximo de $31,2 \mathrm{~m}$. Os dados subdivididos para a validação da equação hipsométrica selecionada obtiveram valor de altura mínima, média e máxima de $21,8 \mathrm{~m}, 25,2 \mathrm{~m}$ e 27,9m; respectivamente. Os diâmetros oscilaram entre 15,8 a 22,1cm, com valor médio de $19,2 \mathrm{~cm}$.

Com a distribuição de frequência dos dados usados no ajuste, em classes de altura, foi possível verificar visualmente maior concentração dos valores à esquerda, característica de assimetria levemente negativa (Figura 1). Ao aplicar o teste de Shapiro-Francia para averiguar a normalidade, constatou-se que os dados seguiram 
distribuição não normal $[\mathrm{W}=0,97086 ; p=0,0148]$, considerando um nível de $5 \%$ de significância.

A ausência de distribuição Normal para variável dependente (h) reforçou a utilização do MLG com distribuição Gamma, o qual é o mais flexível no caso de assimetria quando comparado à distribuição Normal.

FIGURA 1 - Distribuição em porcentagem da altura de eucalipto.

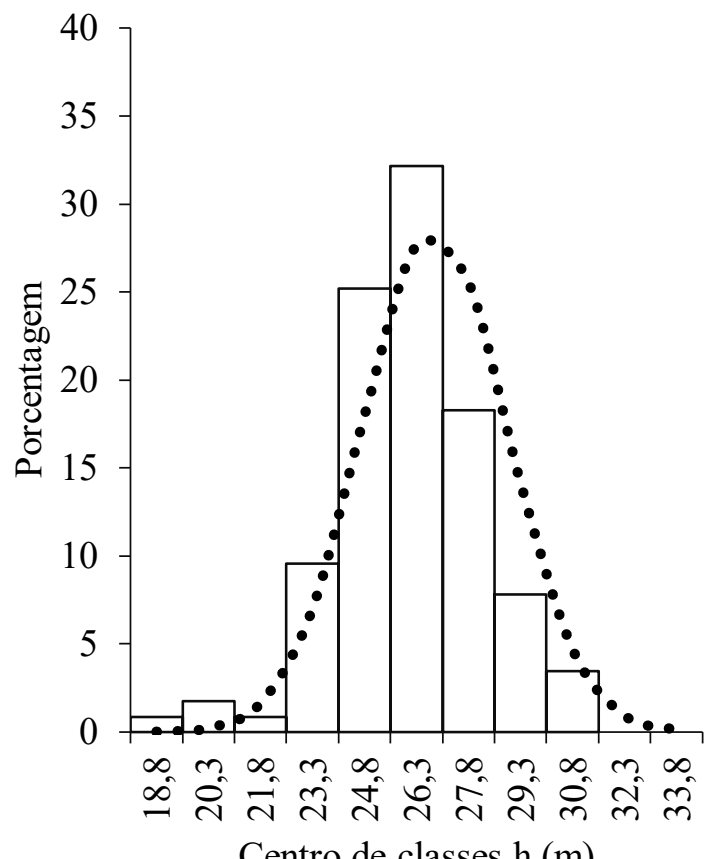

Fonte: autores.

Segundo os critérios estatísticos, a equação 3 [variável independente inversa (1/d)] apresentou a melhor performance (Tabela 1), tanto ao considerar a função de ligação identidade $[\mu]\left(R^{2}=0,367 ; \operatorname{RMSE}=1,64 \mathrm{~m} ; \mathrm{AIC}=115,9\right.$ e $\left.w_{\mathrm{i}}(\mathrm{AIC})=0,24\right)$, quando considerada a função de ligação logarítmica $[\ln (\mu)]\left(R^{2}=0,364 ; \mathrm{RMSE}=\right.$ $1,64 \mathrm{~m} ; \mathrm{AlC}=116,4$ e $\left.w_{\mathrm{i}}(\mathrm{AIC})=0,19\right)$.

O Akaike ponderado $\left[w_{i}(A I C)\right]$ é um estimador relativo usado na comparação entre os modelos, desta maneira, a equação 3 [variável independente inversa - (1/d)] com F.L. identidade $[\mu]$, possui $24 \%$ de probabilidade de ser a equação mais adequada, devido a sua maior porcentagem em relação aos demais testados (Tabela 1).

As equações 1 [variável independente linear - (d)] e 4 [variável independente logarítmica - In (d)] obtiveram performance razoável. Logo, a equação 2 [variável independente quadrática $\left.-\left(\mathrm{d}^{2}\right)\right]$ dentro das F.L. identidade $[\mu]$ e logarítmica $[\ln (\mu)]$, apresentou os piores resultados quando confrontados com os demais analisados (Tabela 1). 
TABELA 1 - Coeficientes estimados e estatísticas de qualidade dos MLG analisados em povoamento de eucalipto.

\begin{tabular}{ccccccccc}
\hline Distribuição & Modelo & F.L. & $\beta_{0}$ & $\beta_{1}$ & $\mathrm{R}^{2}$ & $\mathrm{RMSE}$ & $\mathrm{AIC}$ & $w_{\mathrm{i}}(\mathrm{AIC})$ \\
\hline \multirow{6}{*}{ Gamma } & 1 & & 7,9382 & 0,9507 & 0,358 & 1,65 & 117,6 & 0,11 \\
& 2 & & 16,9448 & 0,0250 & 0,351 & 1,66 & 118,7 & 0,06 \\
& 3 & $\mu$ & 43,8180 & $-336,8670$ & 0,367 & 1,64 & 115,9 & 0,24 \\
& 4 & & $-26,8460$ & 17,9630 & 0,363 & 1,65 & 116,6 & 0,17 \\
& 1 & & 2,5672 & 0,0363 & 0,354 & 1,66 & 118,3 & 0,07 \\
& 2 & & 2,9132 & 0,0009 & 0,347 & 1,67 & 119,5 & 0,04 \\
& 3 & $\ln (\mu)$ & 3,9457 & $-13,0221$ & 0,364 & 1,64 & 116,4 & 0,19 \\
& 4 & & 1,2265 & 0,6902 & 0,360 & 1,65 & 117,2 & 0,12 \\
\hline
\end{tabular}

A tendência estimada para a melhor equação 3 [variável independente inversa (1/d)] com F.L. identidade [ $\mu$ ] usada na descrição da relação hipsométrica de eucalipto no presente estudo pode ser visualizada na Figura (2a). A análise gráfica da distribuição dos resíduos em função das alturas estimadas evidencia uma distribuição satisfatória, com certa proporcionalidade entre as subestimativas e superestimativas (Figura $2 b$ ).

FIGURA 2 - a) Tendência estimada para a descrição da relação hipsométrica em eucalipto; b) Distribuição dos resíduos em porcentagem em função da altura estimada. *Equação 3 [variável independente inversa - (1/d)] com F.L. identidade $[\mu]$.

(a)

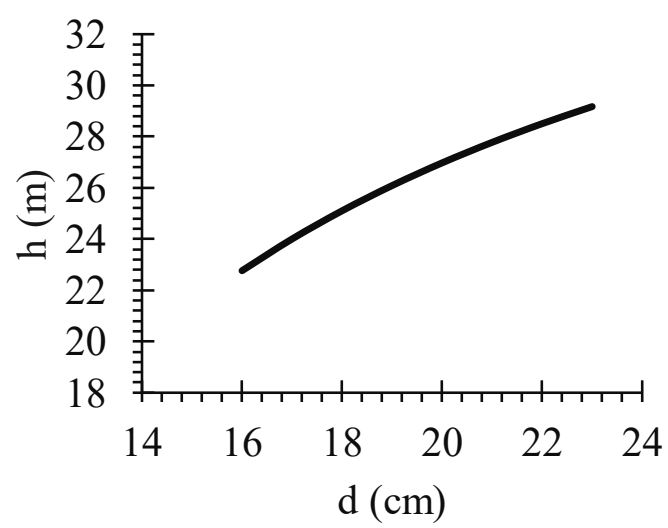

- h estimada (m) (b) Equação 3

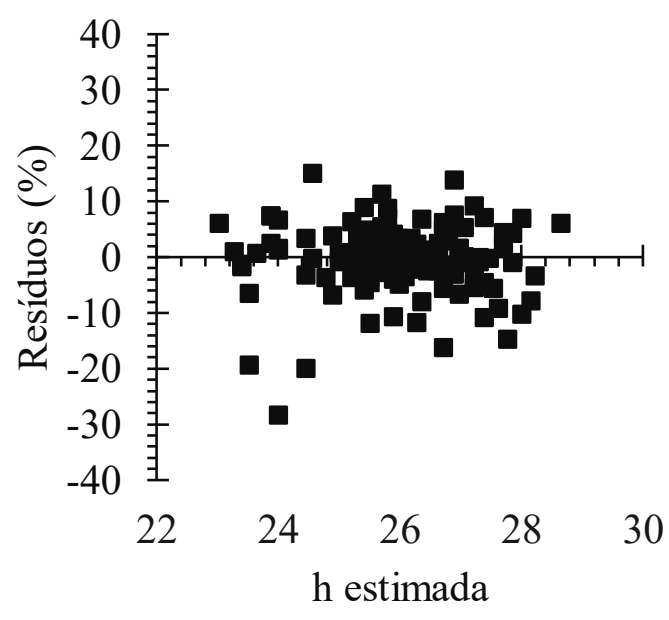

Fonte: autores.

O teste de qui-quadrado $\left(X^{2}\right)$ (Tabela 2 ), realizado para a validação da equação 3 , mostrou não haver diferenças entre os dados observados e estimados. Portanto, o modelo é recomendado para a estimação das alturas de Eucalyptus saligna Smith. no presente estudo. 
TABELA 2 - Teste de $X^{2}$ aplicado nos dados de validação para a melhor equação usada na descrição da relação hipsométrica de eucalipto.

\begin{tabular}{cccc}
\hline Equação & $X^{2}$ calculado & $X^{2}$ tabelado \\
\hline 3 & 4,7544 & ns & $X^{2}[49 ; 1 \%]=74,92$ \\
\hline
\end{tabular}

Em que: $n s=$ não significativo com $(p \leq 0,01)$. ${ }^{*}$ Equação 3 [variável independente inversa - (1/d)] com F.L. identidade [ $\mu]$.

\section{Aplicação}

A equação 3 [variável independente inversa - (1/d)] com F.L. identidade $[\mu]$ foi usada para exemplificar a estimação da altura de uma árvore de eucalipto com $d=$ $21,8 \mathrm{~cm}$.

$$
\mathrm{h}_{\text {estimada }}=[43,8180-336,8670 \times(1 / 21,8)] \approx 28,4 \mathrm{~m} .
$$

\section{DISCUSSÃO}

Na distribuição dos dados foi notável uma leve distribuição assimétrica negativa, verificada através do Teste de Shapiro-Francia, o qual confirma a não normalidade dos dados, corroborando com a utilização de MLG no presente estudo. Visto que, além do potencial de utilização dos MLG para dados com distribuição Normal, em casos de assimetria, a distribuição Gamma possuiu maior flexibilidade tornando a abordagem mais adequada. Caso houvesse a utilização de um modelo linear tradicional seriam necessárias transformações da variável resposta de maneira a assumir uma distribuição Normal.

O estudo de Custódio e Barbin (2009) para predição da sobrevivência de plantas de Eucalyptus grandis detalha uma condição semelhante ao presente estudo, quando os dados apresentaram assimetria, verificada através do teste de Shapiro-Wilk, apresentando não normalidade dos dados, e recorrendo-se ao MLG devido sua maior flexibilidade. Utilizaram-se a distribuição binomial com função de ligação probit obtendo melhor performance.

No estudo de Hess et al. (2015) também foi utilizado MLG com ligação F.L. identidade $[\mu]$ e logarítmica $[\ln (\mu)]$ e distribuições Gamma, Poisson e Normal para estimar o crescimento em altura de Pinus taeda L. A abordagem de MLG utilizando F.L. identidade $[\mu]$ e distribuição Gamma foi capaz de ajustar os dados com precisão, especialmente por não necessitar transformar os dados.

Costa et al. (2016) utilizaram em seu trabalho técnicas de MLG para descrever a relação morfométrica de Araucaria angustifolia, testaram as distribuições Gamma e Normal com F.L. identidade $[\mu]$ e logarítmica $[\ln (\mu)]$. Os autores relataram uma superioridade da distribuição Gamma com F.L identidade [ $\mu$ ] quando comparada as demais abordagens.

Klein et al. (2017) utilizaram MLG para modelar relações morfométricas para Araucaria angustifólia, os autores basearam-se na distribuição Gamma com F.L identidade $[\mu]$ e logarítmica $[\ln (\mu)]$ alcançando performance satisfatória nas predições, obtendo importantes informações para descrever o crescimento da espécie. 


\section{CONCLUSÕES}

A equação 3 [variável independente inversa - (1/d)] com distribuição Gamma e função de ligação identidade $[\mu]$ foi a mais adequada na modelagem da relação hipsométrica de eucalipto para o conjunto de dados analisados.

A técnica de modelos lineares generalizados é importante para a modelagem florestal, principalmente devido a flexibilidade comparada a modelos lineares tradicionais. O seu uso evita a necessidade de transformação de variáveis dependentes e cálculos de erros associados a esse procedimento.

\section{REFERÊNCIAS}

ALVARES, C. A.; STAPE, J. L.; SENTELHAS, P. C.; GONÇALVES, J. L. M.; SPAROVEK, G. Köppen's climate classification map for Brazil. Meteorologische Zeitschrift, v. 22, n. 6, p. 711-728, 2013. doi: 10.1127/0941-2948/2013/0507

BAUTISTA, E. A. L. Modelos lineares mistos e generalizados mistos em estudos de adaptação local e plasticidade fenotípica de Euterpe edulis. Tese (Doutorado em Estatística e Experimentação Agronômica). Universidade de São Paulo. Piracicaba. 2014.

CERQUEIRA, C. L.; MÔRA, R.; TONINI, H.; VENDRUSCOLO, D. G. S.; LANSSANOVA, L. R. et al. Efeito do espaçamento e arranjo de plantio na relação hipsométrica de eucalipto em sistema consorciado de produção. Nativa, v. 7, n. 6, p. 763, 2019. doi: 10.31413/nativa.v7i6.7643

CORDEIRO, G. M.; DEMETRIO, C. G. B. Modelos Lineares Generalizados e Extensões. Piracicaba: USP, p. 302, 2013.

COSTA, E. A.; FINGER, C. A. G.; HESS, A. F. Modelagem do incremento em área transversal de árvores de crescimento livre de Araucaria angustifolia. Revista Brasileira de Biometria, v. 34, n. 3, p. 522-532, 2016. Disponível em:<http://www.biometria.ufla.br/index.php/BBJ/article/view/200>

COSTA, E. A.; FINGER, C. A. G.; CUNHA, T. A. Influência da posição sociológica na relação hipsométrica de Araucaria angustifolia. Revista Brasileira de Ciências Agrárias, v. 9, n. 1, p. 110-116, 2014. doi:10.5039/agraria.v9i1a3201

CUSTÓDIO, T. N.; BARBIN, D. Modelos de predição para sobrevivência de plantas de Eucalyptus grandis. Ciência e Agrotecnologia, v. 33, n. SUPPL., p. 1948-1952, 2009. doi: $10.1590 / S 1413-70542009000700043$

FAO - Food and Agriculture Organization of the United Nations - Global Forest Resources Assessment 2015: How are the world's forests changing?. 2015. Disponível em: <http://www.fao.org/3/a-i4793e.pdf> Acesso em: 2020.

FOX, J. Applied Regression Analysis and Generalized Linear Models. 2. ed. California: Sage Publications. p. 816, 2015.

HESS, A. F.; CIANORSCHI, L.; SILVESTRE, R.; SCARIOT, R.; RICKEN, P. Aplicação dos modelos lineares generalizados para estimativa do crescimento em altura. Pesquisa Florestal Brasileira, v. $35, \quad$ n. $84, \quad$ p. $427,2015$. doi: https://doi.org/10.4336/2015.pfb.35.84.604 
IBÁ - Indústria Brasileira de árvores - Relatório 2017. Indústria Brasileira de Árvores - IBÁ, 2017. Disponível em: <https://www.iba.org/datafiles/publicacoes/cenarios/61cenarios.pdf> Acesso em: 2020.

IBGE - Instituto Brasileiro de Geografia e Estatística - Produção da extração vegetal e da silvicultura 2015. 2016. Disponível em:<https://www.ibge.gov.br/estatisticasnovoportal/economicas/agricultura-epecuaria/9105-producao-da-extracao-vegetal-eda-silvicultura.html?\&t=resultados> Acesso em: 2020.

KLEIN, D. R.; HESS, A. F.; KREFTA, S. M.; VIEIRA FILHO, M. D. H.; CIARNOSCKI, L. D. et al. Relações morfométricas para Araucaria angustifolia (Bertol.) Kuntze em Santa Catarina. Floresta, v. 47, n. 4, p. 501-512, 2017. doi: 10.5380/rf.v47i4.49667

LINDSEY, J. K. Applying generalized linear models. New York: Springer, p. 256, 1997.

MICROSOFT CORPORATION. Microsoft Excel 2019. 2018. Disponível em: $<$ https://office.microsoft.com/excel>. Acesso em: 2020.

MOSER. P.; OLIVEIRA, L. Z. Regressão aplicada à dendrometria: Uma introdução e iniciação à linguagem R. 1. ed. Blumenau: Edifurb, p. 152, 2017.

MÜLLER, M. Generalized linear models. Journal of the Royal Statistical Society, v. 135 , n. 3, p. $681-709$, 2012. doi: $10.2307 / 2344614$

NICOLETTI, M. F.; SOUZA, K.; SILVESTRE, R.; FRANÇA, M. C.; ROLIM, F. A. Relação hipsométrica para Pinus taeda L. em diferentes fases do ciclo de corte. Floresta e Ambiente, v. 23, n. 1, p. 80-89, 2016. doi: 10.1590/2179-8087.051513.

R Core Team. R: A language and environment for statistical computing. R Foundation for Statistical Computing, Vienna, Austria, 2020. Disponível em: <https://www.Rproject.org/>.

RETSLAFF, F. A. S.; FIGUEIREDO FILHO, A.; DIAS, A. N.; BERNETT, L. G.; FIGURA, M. A. Curvas de sítio e relações hipsométricas para Eucalyptus grandis na região dos Campos Gerais, Paraná. Cerne, v. 21, n. 2, p. 219-225, 2015. doi: 10.1590/01047760201521021349

RIBEIRO, A.; FERRAZ FILHO, A. C.; MELLO, J. M.; FERREIRA, M. Z.; LISBOA, P. $M$. M. et al. Estratégias e metodologias de ajuste de modelos hipsométricos em plantios de Eucalyptus sp. Cerne, v. 16, n. 1, p. 22-31, 2010. doi: 10.1590/S010477602010000100003

SANQUETTA, C. R.; BEHLING, A.; CORTE, A. P. D.; RUZA M. S.; SIMON A. et al. Relação hipsométrica em inventários pré-corte em povoamentos de Acacia mearnsii De Wild. Científica, v. 42, n. 1, p. 80-90, 2014 . doi: 10.15361/19845529.2014v42n1p80-90

SOARES, K. L.; COSTA, L. S.; ARAÚJO, M. S.; CALIXTO JÚNIOR, J. E. D. Modelagem hipsométrica de Eucalyptus grandis W. Hill ex Maiden. no sudeste do estado de Goiás. Journal of Neotropical Agriculture, v. 4, n. 5, p. 51-58, 2017. doi: 10.32404/rean.v4i5.2184 